\title{
Construção da Tarefa de Leitura de Palavras e Pseudopalavras (TLPP) e Desempenho de Leitores Proficientes
}

\author{
Jaqueline de Carvalho Rodrigues ${ }^{1}$ \\ Programa de Pós Graduação em Psicologia da Universidade Federal do Rio Grande do Sul, \\ Porto Alegre, RS, Brasil \\ Núcleo de Estudos em Neuropsicologia Cognitiva, Porto Alegre, RS, Brasil \\ Alexandre de Pontes Nobre \\ Programa de Pós Graduação em Psicologia da Universidade Federal do Rio Grande do Sul, \\ Porto Alegre, RS, Brasil \\ Laboratório de Biossinais em Fenomenologia e Cognição, Porto Alegre, RS, Brasil \\ Laboratório de Fenomenologia Experimental e Cognição, Porto Alegre, RS, Brasil \\ Gustavo Gauer \\ Departamento de Psicologia do Desenvolvimento e da Personalidade, \\ Porto Alegre, RS, Brasil \\ Coordenação do Laboratório de Biossinais em Fenomenologia e Cognição, \\ Porto Alegre, RS, Brasil \\ Jerusa Fumagalli de Salles \\ Departamento de Psicologia do Desenvolvimento e da Personalidade da Universidade \\ Federal do Rio Grande do Sul, Porto Alegre, RS, Brasil \\ Coordenação do Núcleo de Estudos em Neuropsicologia Cognitiva, Porto Alegre, RS, Brasil
}

\section{Resumo}

Estímulos em tarefas de avaliação neuropsicológica da leitura de palavras devem ser sensíveis aos efeitos psicolinguísticos e aos tipos de erros que indiquem processamentos falhos e preservados. O objetivo deste estudo foi apresentar o processo de construção de uma tarefa de leitura de palavras e pseudopalavras e avaliar o desempenho de leitores proficientes. Foram utilizados estímulos selecionados de listas de palavras, considerando os critérios de frequência, extensão, lexicalidade e regularidade, que passaram por um processo de análise entre três juízes. Para a avaliação do desempenho, participaram 35 crianças $(M=10,80 ; D P=0,83$ anos) de escolas particulares de Porto Alegre e 54 adultos, divididos em dois grupos de escolaridade: até 10 anos de estudo $(n=25)$ e 11 ou mais anos de estudo $(n=29)$. Observaram-se os efeitos psicolinguísticos de lexicalidade, frequência, extensão e regularidade, indicando que os estímulos da tarefa eram apropriados. O desempenho na tarefa correlacionou-se significativa e positivamente com escolaridade e hábitos de leitura e escrita. Na comparação entre grupos, observou-se que os adultos com menor escolaridade tiveram desempenho inferior ao dos adultos com maior escolaridade e das crianças. Além de ressaltar a influência da idade e da escolaridade dos participantes, os dados apresentados podem auxiliar clínicos que desejam utilizar essa tarefa em uma avaliação mais aprofundada da leitura de palavras e pseudopalavras.

Palavras-chave: Leitura, avaliação neuropsicológica, adultos, crianças, modelo de dupla-rota.

Endereço para correspondência: Universidade Federal do Rio Grande do Sul, Instituto de Psicologia, Núcleo de Estudos em Neuropsicologia Cognitiva, Rua Ramiro Barcelos, 2600, sala 114, Santa Cecília, Porto Alegre, RS, Brasil 90035-003. Fone: (51) 3308-5341. E-mail: jaquecarvalhorodrigues@gmail.com, alpnobre@gmail. com,gusgauer@gmail.com e jerusafsalles@gmail.com 


\title{
Construction of a Word and Pseudoword Reading Task (TLPP) and Performance of Proficient Readers
}

\begin{abstract}
Stimuli in tasks for neuropsychological assessment of word reading should be sensitive to psycholinguistic effects and to types of errors which may indicate impaired and preserved abilities in reading. The aim of this study was to present the process of construction of a word and pseudoword reading task for adults and evaluate the performance of proficient readers in the task. Stimuli were selected from word lists, considering the criteria of frequency, extension, lexicality and regularity, and underwent an evaluation process between judges. For the assessment of performance, 35 children $(M=10.80 ; S D=$ .83 years) from private schools in Porto Alegre, Brazil, and 54 adults, divided in two groups: 10 or less years of study $(n=25)$ and 11 or more years of study $(n=29)$. The psycholinguistic effects of lexicality, frequency, extension and regularity were observed, indicating that the stimuli were appropriate. Performance in the tasks correlated significantly and positively with education and reading and writing habits. In the comparison between groups, adults with lower education exhibited lower performance compared to adults with higher education and to children. Besides highlighting the influence of age and education of the participants, the data may help clinicians who wish to use this task in a more detailed assessment of word reading.
\end{abstract}

Keywords: Reading, neuropsychological assessment, adults, children, dual-route model.

\section{Construcción de la Tarea de Lectura de Palabras y Pseudopalabras (TLPP) y el Rendimiento de Lectores Competentes}

\section{Resumen}

Los estímulos en las tareas de evaluación neuropsicológica de la lectura de palabras deben ser sensibles a los efectos psicolinguísticos y a los tipos de errores que indican procesamientos deficientes y conservados. El objectivo de este estudio fue presentar el proceso de construcción de una tarea de lectura de palabras y pseudopalabras y el desempeño de lectores proficientes. Se utilizó estímulos seleccionados de listas de palabras, teniendo en cuenta los criterios de frecuencia, extensión, lexicality y regularidad, que han sido sometidos a un proceso de análisis entre tres jueces. Para la evaluación del desempeño, participaron 35 niños $(M=10.80 ; S D=.83$ años $)$ de escuelas privadas de Porto Alegre, Brasil, y 54 adultos, divididos en dos grupos de escolaridad: hasta 10 años de estudio $(n=25)$ y 11 o más años de estudio $(n=29)$. Se observaron efectos de lexicalidad, frecuencia, extensión y regularidad, lo que indica que los estímulos de la tarea eran adecuados. El rendimiento de la tarea se correlacionó significativa y positivamente con escolaridad y hábitos de lectura y escritura. En la comparación entre grupos, fue observado que el grupo de adultos con menor escolaridad ha tenido desempeño inferior al grupo de adultos con mayor escolaridad e a los niños. Además de enfatizar la influencia de la edad e de la escolaridad de los participantes, los datos que se presentan pueden ayudar a los clínicos que desean utilizar esta tarea en una evaluación más a fondo de la lectura de palabras.

Palabras clave: Lectura, evaluación neuropsicológica, adultos, niños, modelo de doble-ruta.

A leitura de palavras é uma habilidade fundamental no cotidiano da maioria das pessoas. Para ler adequadamente é necessário identificar a combinação dos códigos visuais como letras (representações ortográficas) e a sua pronúncia (decodificação fonológica), e compreender o significado do que está sendo lido (processamento semântico; Salles \& Parente, 2004). Défi- 
cits em uma ou mais dessas capacidades podem contribuir para as dificuldades de leitura, como aquelas conhecidas como dislexias ou alexias.

As dislexias podem ocorrer desde a infância, prejudicando o aprendizado da leitura, sendo caracterizadas como dislexias do desenvolvimento, ou podem ocorrer como consequência de algum dano cerebral, ocasionando as dislexias adquiridas (Ellis, 1995). As dislexias do desenvolvimento, identificadas em crianças, acometem cerca de 5\% da população mundial (Thambirajah, 2010), com resultados semelhantes para a população brasileira (Gutierrez \& Tomasi, 2011). Já as dislexias adquiridas, que ocorrem geralmente em adultos, apresentam-se após algum tipo de lesão, como o acidente vascular cerebral, sendo ainda subdiagnosticadas (Sinanović, Mrkonjić, Zukić, Vidović, \& Imamović, 2011). O aprimoramento da avaliação de possíveis transtornos de leitura, visando posteriores intervenções, mostra-se importante e demanda instrumentos adequados.

No Brasil, diversas tarefas têm sido desenvolvidas desde a década de 1990 para avaliar a leitura de palavras, a partir de uma abordagem cognitiva (ver, por exemplo, Capovilla \& Capovilla, 1998; Capovilla, Capovilla, \& Macedo, 1998; Lúcio, Moura, Nascimento, \& Pinheiro, 2012; Salles \& Parente, 2002). Entretanto, a maioria dessas tarefas é direcionada à avaliação de crianças, especialmente em fase de alfabetização. Por serem extraídas de listas baseadas em livros infantis, as palavras utilizadas nessas tarefas costumam ser demasiadamente fáceis para crianças já alfabetizadas, adolescentes e adultos. Assim, há necessidade de construir um instrumento para avaliar a leitura dessa população de maior idade. Este estudo apresenta o processo de construção de uma tarefa para avaliar a leitura de palavras e pseudopalavras e o desempenho quantitativo e qualitativo de crianças em anos escolares mais avançados e adultos.

A tarefa foi elaborada com base no modelo de dupla-rota, que possibilita uma explicação satisfatória para o desempenho e também para uma série de prejuízos na leitura de palavras (Coltheart, 2006; Pritchard, Coltheart, Palethorpe, \& Castles, 2012) e tem sido amplamente utilizado na literatura brasileira (ver, por exemplo, Capo- villa, Varanda, \& Capovilla, 2006; Rodrigues \& Salles, 2013; Salles, Piccolo, Zamo, \& Toazza, 2013). Segundo esse modelo, a leitura pode ser realizada por meio de dois mecanismos denominados "rotas", sendo uma fonológica e outra lexical (Coltheart, 2006).

Quando uma palavra é lida, é inicialmente decodificada nos traços e letras que a compõem e em seguida é processada por uma das rotas ou por ambas. Enquanto a rota fonológica converte grafemas (letras) em fonemas (sons), a rota lexical é constituída por duas subrotas: uma rota lexical semântica (que acessa o significado da palavra) e outra não-semântica. Ambas as subrotas identificam a palavra como um todo, através de léxicos ortográfico e fonológico e, no caso da rota lexical semântica, de um sistema semântico. Após o processamento e identificação da palavra por alguma dessas duas rotas, um output fonológico é produzido (Coltheart, 2006).

No modelo de dupla-rota, a rota fonológica não permite leitura eficiente de palavras irregulares, enquanto a rota lexical pode identificar corretamente apenas palavras reais, mas não pseudopalavras. Dessa forma, o modelo prevê, corretamente, que danos seletivos às rotas podem causar prejuízos na leitura de estímulos específicos, de acordo com o tipo de palavra lida (Pritchard et al., 2012). A partir da análise de quais palavras o participante frequentemente erra, podem-se verificar os efeitos psicolinguísticos, tais como regularidade (melhor desempenho na leitura das palavras regulares, comparado às irregulares), lexicalidade (desempenho superior na leitura de palavras reais, comparado às pseudopalavras), frequência (maior número de acertos na leitura de palavras frequentes, quando comparado às não frequentes) e extensão (melhor desempenho na leitura de palavras curtas, em comparação às longas). Efeitos de lexicalidade e frequência podem sugerir uma estratégia de leitura prioritariamente lexical, enquanto os efeitos de extensão e regularidade indicam uso prioritário da rota fonológica na leitura de palavras. Assim, é importante manipular as características psicolinguísticas dos estímulos e analisar detalhadamente os erros cometidos pelos participantes, uma vez que essas informações 
são importantes para verificar os mecanismos de leitura mais utilizados (Pinheiro, Cunha, \& Lúcio, 2008).

Nesse contexto, os objetivos desse trabalho foram (a) apresentar as etapas de construção de uma tarefa de leitura de palavras e pseudopalavras, (b) mostrar a relação entre os escores obtidos e as demais variáveis estudadas (idade, anos de estudo, hábitos de leitura e escrita e QI) em cada grupo e (c) descrever o desempenho de adultos e crianças na tarefa de leitura (número de acertos, de erros e a frequência de tipos de erros), disponibilizando dados preliminares. Espera-se que esse estudo possa contribuir com a avaliação da leitura de palavras e das dislexias adquiridas e do desenvolvimento, de acordo com a abordagem da Neuropsicologia Cognitiva.

\section{Método}

\section{Participantes}

Participaram do processo de construção da Tarefa de Leitura de Palavras e Pseudopalavras (TLPP) três professores e pesquisadores, doutores na área de neuropsicologia e linguagem, com conhecimento em linguística e experiência clínica na avaliação das dificuldades de leitura. Depois de concluída a construção, a tarefa foi aplicada em 35 crianças e 54 adultos, conforme a Tabela 1. Esse estudo foi aprovado pelo Comitê de Ética em Pesquisa do Instituto de Psicologia da Universidade Federal do Rio Grande do Sul (UFRGS; número de parecer 31477) e todos os participantes ou seus responsáveis assinaram um Termo de Consentimento Livre e Esclarecido.

Tabela 1

Número de Participantes por Sexo, Média e Desvio-Padrão da Idade e Anos de Estudo das Crianças e dos Adultos

\begin{tabular}{lccc}
\hline & $\begin{array}{c}\text { Crianças } \\
(n=35)\end{array}$ & $\begin{array}{c}\text { Adultos até 10 anos } \\
\text { de estudo } \\
(n=25)\end{array}$ & $\begin{array}{c}\text { Adultos com 11 ou mais } \\
\text { anos de estudo } \\
(n=29)\end{array}$ \\
\hline Sexo (F/ M) & $18 / 17$ & $15 / 10$ & $16 / 13$ \\
Idade & $10,80(0,83)$ & $54,80(10,15)$ & $52,62(8,93)$ \\
Anos de estudo & $4,63(0,49)$ & $7,08(1,89)$ & $13,63(2,70)$ \\
Hábitos de Leitura e Escrita* & & $9,32(3,71)$ & $15,59(5,18)$ \\
\hline
\end{tabular}

Nota. $\mathrm{M}$ = masculino; $\mathrm{F}$ = feminino; *escore dos Hábitos de Leitura e Escrita variavam de zero a 32 pontos de acordo com um questionário utilizado para verificar sua frequência semanal (Pawlowski et al., 2012).

As crianças cursavam o quinto e o sexto ano escolar (10 a 13 anos de idade) em escolas particulares de Porto Alegre, RS, contatadas por conveniência. As turmas foram indicadas por um profissional da escola e as crianças eram indicadas pela professora. Foram selecionadas aquelas sem histórico de repetência escolar ou diagnóstico de doenças neurológicas e/ ou psiquiátricas relatadas por seu responsável. Além disso, deveriam obter desempenho igual ou superior ao percentil 25 nas Matrizes Progressivas Coloridas de Raven - Escala Especial (Angelini, Alves, Custódio, Duarte, \& Duarte, 1999).

Os adultos faziam parte da comunidade em geral, sendo divididos em dois grupos por esco- laridade, após se verificar, por meio de comparação de médias, que até 10 anos de estudo não havia diferenças nos desempenhos na TLPP. Os grupos de adultos não se diferenciaram quanto à idade $(p=0,70)$, somente em relação à escolaridade $(p<0,001)$, conforme a divisão previamente realizada. Ademais, os adultos com maior número de anos estudados apresentavam maiores hábitos de leitura e escrita $(p<0,001)$ do que o grupo com até 10 anos de estudo, mensurados com um questionário específico sobre a frequência semanal destes hábitos (Pawlowski et al., 2012). Os indivíduos apresentavam no mínimo quatro anos de estudo formal, nenhum histórico de doenças neurológicas e/ ou psiquiátricas 
autorrelatados, ausência de sintomas depressivos de acordo com o Inventário Beck de Depressão (BDI-II) (Beck, Steer, \& Brown, 1996; Gorenstein, Pang, Argimon, \& Werlang, 2011) e desempenho adequado para sua idade e escolaridade no Mini Exame do Estado Mental (Chaves \& Izquierdo, 1992; Kochhann, Varela, Lisboa, $\&$ Chaves, 2010).

\section{Procedimentos de Construção da TLPP}

O processo de construção da TLPP seguiu cinco etapas, semelhantes àquelas da Tarefa de Escrita de Palavras/pseudopalavras sob ditado (Rodrigues \& Salles, 2013): (a) Escolha dos critérios psicolinguísticos, (b) Seleção dos itens a partir de listas de palavras, (c) Análise da primeira versão da tarefa por dois juízes especialistas, (d) Reformulações da tarefa e análise por um terceiro juiz, (e) Versão final da TLPP.

Escolha dos Critérios Psicolinguísticos. Optou-se por controlar as variáveis classe gramatical e concretude (imageabilidade), sendo incluídos somente substantivos comuns, simples e concretos. Foram manipuladas as variáveis extensão, frequência, lexicalidade e regularidade dos estímulos.

Como critério de extensão, foram selecionadas palavras curtas com até duas sílabas (ou até cinco fonemas) e palavras longas com três ou mais sílabas (acima de seis fonemas; Salles \& Parente, 2007). Em relação à frequência, foram consideradas frequentes palavras com número de ocorrências igual ou superior a 1186 (valor representativo das 3000 palavras mais frequentes da língua Portuguesa, de um total de 255035 palavras), e não frequentes palavras com ocorrência menor ou igual a 300, de acordo com a lista publicada por Sardinha (2003), extraídas de materiais escritos da internet, jornais e revistas. Para controlar a imageabilidade dos estímulos, foram incluídas nessa tarefa somente palavras concretas, de acordo com a lista publicada por Janczura, Castilho, Rocha, Van Erven e Huang (2007).

Para verificar o efeito de lexicalidade, foram incluídas na tarefa pseudopalavras e palavras reais. As pseudopalavras foram criadas a partir de palavras reais, que tiveram suas letras e/ou sílabas invertidas, substituídas ou omitidas, mantendo combinações que não existem no léxico da língua, mas que possuem a estrutura de palavras aceitas no português (Salles \& Parente, 2007). As palavras reais foram organizadas quanto à sua regularidade e irregularidade para a leitura, de acordo com Pinheiro (2003). São regulares para leitura as palavras em que o som das letras na conversão grafema-fonema possui uma correspondência unívoca, ou seja, há apenas uma forma de ler as palavras de acordo com as regras ortográficas. Há apenas três situações em que as palavras do português são consideradas irregulares para a leitura: com a consoante $<x>$ e com as vogais $<\mathrm{e}>\mathrm{e}<\mathrm{0}>$. A consoante $<\mathrm{x}>$ pode ser associada, independentemente da posição, aos fonemas $/ \mathrm{S} /, / \mathrm{s} /, / \mathrm{ks} /$ ou $/ \mathrm{z} /$, enquanto que as vogais $<\mathrm{e}>\mathrm{e}<_{\mathrm{O}}>$, quando estão em posição tônica em palavras paroxítonas, são consideradas ambíguas, pois podem remeter aos fonemas /e/ ou $/ \epsilon /$ e $/ \mathrm{o} / \mathrm{ou} / \mathrm{o} /$.

Seleção dos Itens a partir de Listas de Palavras. Foram selecionadas palavras concretas da lista publicada por Janczura et al. (2007) e destas escolheram-se as que apresentavam alta e baixa frequência de ocorrência (leitura), de acordo com Sardinha (2003). Incluíram-se na primeira versão da tarefa 32 palavras, sendo 16 regulares (ou regidas por regra), 16 irregulares e 16 pseudopalavras. Todos esses estímulos foram ainda agrupados em curtos e longos. A TLPP foi então enviada a dois juízes.

Análise da Primeira Versão da Tarefa por Dois Juizes Especialistas. O primeiro juiz indicou que "assinatura" não seria representativa de palavra frequente, enquanto que "galinha", "dente", "sofá" e "barba" não poderiam ser consideradas palavras não frequentes. Ainda, orientou que a palavra "homem" que estava entre os estímulos longos, na verdade, teria que estar entre os curtos.

O segundo juiz concordou com o primeiro em todas as ponderações, exceto que a palavra "barba" poderia ser apreciada como não frequente e acrescentou que "boneca" também não seria adequadamente classificada nesse conjunto. 
Além disso, este juiz sugeriu que fosse aumentado o número de itens da TLPP, visto que para verificar os efeitos psicolinguísticos de regularidade e frequência na leitura faz-se necessário ter um número significativo de palavras para poder identificá-los nas análises estatísticas.

Reformulações da Tarefa e Análise por um Terceiro Juiz. Seguiram-se as orientações de ambos os juízes, uma vez que estas se mostraram pertinentes para melhorar a tarefa. Substituíram-se as palavras destacadas e incluiu-se uma palavra a mais em cada agrupamento, aumentando de 16 para 24 itens, a fim de melhor verificar os efeitos psicolinguísticos de frequência e regularidade. A segunda versão da TLPP foi enviada a um novo juiz que a considerou apropriada, não identificando nenhuma inadequação nos itens ou na quantidade de estímulos.

Versão Final da TLPP. Após todas essas etapas, a TLPP foi concluída com 24 palavras regulares, 24 irregulares e 24 pseudopalavras. Cada agrupamento foi organizado em 12 estímulos curtos e 12 longos. Havia ainda 24 palavras frequentes e 24 não frequentes. No total, a TLPP apresenta 72 estímulos (48 palavras e 24 pseudopalavras; Anexo A).

\section{Procedimentos de Aplicação da TLPP}

A TLPP foi administrada individualmente em local apropriado. Inicialmente, as instruções da TLPP eram lidas ao participante e, em seguida, os estímulos eram mostrados em ordem sequencial em um livro impresso em fonte Arial, cor preta, tamanho 24. As respostas eram gravadas em arquivo de áudio e posteriormente ouvidas para correção. O participante poderia corrigir-se, sendo que o avaliador informava que isso era permitido.

\section{Procedimentos de Pontuação da TLPP}

Análise Quantitativa do Número de Acertos na Tarefa. Os escores obtidos pelos participantes foram contabilizados de três maneiras distintas: número de respostas corretas (aceitando-se as auto-correções), análise qualitativa dos erros (número total de erros e dos tipos de erros) e efeitos psicolinguísticos (regularidade, frequência, extensão e lexicalidade). Inicialmente, identificou-se o número de acertos na TLPP, conforme a Tabela 2. Todos esses escores foram convertidos para porcentagem para em seguida serem verificados os efeitos psicolinguísticos.

Análise dos Efeitos Psicolinguísticos na Tarefa. $\mathrm{O}$ efeito de frequência era identificado pela subtração da porcentagem de acertos das palavras frequentes pelas não frequentes. $\mathrm{O}$ efeito de regularidade era contabilizado pelas porcentagens de palavras regulares menos as irregulares corretamente lidas. O efeito de extensão foi calculado diminuindo-se o valor das porcentagens de acertos das palavras curtas pelas longas, sendo uma medida obtida para as palavras reais e outra para as pseudopalavras. Por fim, o efeito de lexicalidade era verificado a partir das porcentagens de palavras reais menos a porcentagem de pseudopalavras lidas corretamente.

Análise dos Tipos de Erros Cometidos na Tarefa. Análises de erros foram conduzidas para cada item (podendo conter um ou mais tipos de erros) lido incorretamente pelo menos uma vez, mesmo seguido de autocorreção. A análise dos erros foi conduzida por dois juízes previamente treinados, que ouviram todas as gravações e contabilizaram os tipos de erros, não havendo discrepâncias entre suas classificações. Esses erros foram agrupados em um protocolo de correção, conforme a Tabela 3.

\section{Análise dos Dados}

Inicialmente, a normalidade dos dados foi verificada através do teste de Kolmogorov-Smirnov, constatando-se uma distribuição não normal $(p<0,001)$. Para investigar quais variáveis poderiam estar relacionadas com o desempenho dos grupos na TLPP, foram conduzidas correlações de Spearman, analisando as correlações das porcentagens de acertos com anos de estudo, idade, QI (para as crianças) e hábitos de leitura e escrita (para os adultos). Após ser verificado quais variáveis poderiam estar relacionadas com os escores dos participantes na TLPP, analisou-se o desempenho quantitativo comparando-se o desempenho entre os grupos 


\section{Tabela 2}

Distribuição das Palavras da TLPP e Pontuações

\begin{tabular}{|c|c|c|c|c|}
\hline & \multicolumn{2}{|c|}{ Regular/Regra } & \multicolumn{2}{|c|}{ Irregular } \\
\hline & \multicolumn{2}{|c|}{ Total: $\quad / 24$} & \multicolumn{2}{|c|}{ Total: ___ $/ 24$} \\
\hline & Longa & Curta & Longa & Curta \\
\hline & Total: & Total: ___ $/ 12$ & Total: & Total: ___ $/ 12$ \\
\hline \multirow[b]{3}{*}{ Frequente } & Dinheiro & Filho & Escola & Terra \\
\hline & Família & $\underline{\text { Carta }}$ & Exército & Droga \\
\hline & Criança & $\underline{\text { Leite }}$ & Transporte & Sexo \\
\hline \multirow{5}{*}{ Total: $\_$_ $/ 24$} & $\underline{\text { Cidade }}$ & $\underline{\text { Cama }}$ & Caderno & Festa \\
\hline & Futebol & $\underline{\text { Rede }}$ & Janela & Jovem \\
\hline & Comida & Meia & Amarelo & Rosa \\
\hline & Machucado & Barba & Acerola & Gola \\
\hline & Tabaco & Grade & Chinelo & Tosse \\
\hline \multirow{8}{*}{$\begin{array}{l}\text { Não frequente } \\
\text { Total: } ـ \quad / 24\end{array}$} & Fermento & Lesma & Saxofone & Selva \\
\hline & Correnteza & Jaula & Taxímetro & Gosma \\
\hline & Felino & Cárie & Farelo & Terno \\
\hline & Picada & $\underline{\text { Garra }}$ & Insêto & Torta \\
\hline & Longa & Curta & Longa & Curta \\
\hline & Total: & Total: ___ 12 & Total: $\_/ 12$ & Total: $\_$_ 12 \\
\hline & Divairo & Tilhu & Maralo & Lajau \\
\hline & Etixero & Varte & Chunile & Senjo \\
\hline \multirow{4}{*}{$\begin{array}{l}\text { Pseudopalavras } \\
\text { Total: } \_ \text {_ } 24\end{array}$} & Taspobe & Teile & Fosaxone & Gadra \\
\hline & Cavermo & Bafau & Zarronte & Moxe \\
\hline & Jenala & Zareu & Tomenfo & Nurto \\
\hline & Rorola & Tisso & Bolefu & Mesla \\
\hline
\end{tabular}

Nota. As regras do Português e as irregularidades nas palavras estão sublinhadas.

através do teste de Kruskal-Wallis. Realizou-se também análise intragrupo, comparando-se o desempenho dos participantes no grupo de estímulos de acordo com a regularidade, lexicalidade, frequência e extensão das palavras. Em seguida, foram elaborados os dados preliminares na TLPP, utilizando-se os percentis (porcentagem de acertos em palavras reais, regulares, irregulares, pseudopalavras e no total da tarefa), uma vez que o desempenho dos participantes não seguiu uma distribuição normal.

\section{Resultados}

Em relação ao desenvolvimento da TLPP, os estímulos foram distribuídos em um protocolo de aplicação em palavras e pseudopa- lavras, com instruções específicas para cada (Anexo A). A organização da tarefa quanto à regularidade/ regra e irregularidade encontra-se na Tabela 2.

Identificaram-se correlações significativas positivas de fraca a moderada entre as medidas da TLPP e as variáveis anos de estudo, idade e hábitos de leitura e escrita dos participantes (Tabela 4). Ressalta-se que no grupo de crianças as variáveis idade e anos de estudo estão positiva e fortemente correlacionadas $(\rho=0,73 ; p<0,01)$. $\mathrm{O}$ QI não mostrou correlacionar-se com nenhuma das variáveis da TLPP. Nos adultos, hábitos de leitura e escrita e escolaridade apresentavam correlação positiva e forte entre si $(\rho=0,65 ; p$ $<0,01)$. A variável idade não se correlacionou significativamente com nenhuma das medidas da TLPP. 
Tabela 3

Descrição dos Tipos de Erros na Leitura de Palavras e Pseudopalavras

\begin{tabular}{ccc}
\hline Tipo de erro & Descrição & Exemplo
\end{tabular}

Paralexias

Fonológica

Semântica

Semântico-fonológica

Morfêmica

Verbal

Desconsideração

de regras

contextuais

Regularização

Acentuação

Lexicalização

Neologismo

Substituição, Omissão, Adição e Transposição

Perseveração
Ler como uma palavra fonologicamente semelhante ao estímulo alvo, mas ocorrendo adições, omissões, substituições ou transposições de sons. Mantêm-se pelo menos a metade do estímulo, e este sempre existe no português.

Leitura de uma palavra que é semanticamente relacionada ao estímulo, mas formalmente distinta deste. O produto final é sempre uma palavra do português.

Leitura de uma palavra que existe no português, semântica e fonologicamente semelhante ao estímulo, mantendo pelo menos $50 \%$ da palavra original. Podem ocorrer adições, omissões, substituições ou transposições de sons.

Leitura de uma palavra distinta do estímulo escrito, mas com alguns morfemas semelhantes, mantendo ao menos metade da palavra original. Mudam-se os sufixos, prefixos ou a raiz da palavra, que sempre é existente no português.

Leitura de uma palavra distinta do estímulo, sem relação semântica ou formal com este. A palavra lida existe no português, mas apresenta mais da metade dos fonemas ou grafemas diferentes da original.

Leitura com substituição ou omissão de sons de letras ou conjuntos de letras cuja correspondência grafofonêmica é regulada por regras contextuais. Também estão incluídas nesta categoria dificuldades em ler corretamente sons nasais.

Substituição, em palavras irregulares, de um ou mais sons por outro(s) que potencialmente representam a mesma letra ou grupo de letras.

Erros na pronúncia da sílaba tônica ou consideração incorreta de acentos gráficos.

Leitura de uma pseudopalavra como uma palavra real que possui uma semelhança formal com o estímulo. O produto é uma palavra que mantém $50 \%$ ou mais de sua estrutura.

Leitura de uma pseudopalavra em substituição a uma palavra ou uma pseudopalavra distinta do estímulo. $\mathrm{O}$ alvo é mais do que $50 \%$ distinto deste.

Leitura de palavras ou pseudopalavras com substituição, omissão, adição ou transposição de sons, tendo como produto final uma pseudopalavra que mantém $50 \%$ ou mais da estrutura do estímulo.

Leitura repetida de uma palavra ou pseudopalavras inteira, ou parte dela, apresentada anteriormente, em substituição ao estímulo-alvo, ou perseveração de erro. /'k $\widetilde{x} n$ e /

para $<$ cama $>$

/fa' rin $\widetilde{\boldsymbol{v}} /$ para $<$ fermento $>$

/'Ro $\int \mathrm{u} /$

para $<$ rosa $>$

/kar'tejৎu/

para $<$ carta $>$

/'sou/

para $<$ cárie $>$

/'Cassa/ para $<$ casa $>$,

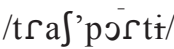
para $<$ transporte $>$

/saSo'font/ para $<$ saxofone $>$

/ezer'situ/ para $<$ exército $>$

/sakso'fonit/ para $<$ fosaxone $>$

/por'Kar/ para $<$ zarronte $>$

/ze'nele/ para <jenala $>$,

/'letí/ para <leite $>$, /'sẽzuS/ para $<$ senjo $>$, / ala'me $r \mathrm{u} /$ para $<$ amarelo $>$

/'Rue/ repetidas vezes, ou "inicia" /Ru/, autocorrigese e inicia outra palavra. 
Tabela 4

Matriz de Correlações entre as Variáveis Mensuradas na TLPP e as Variáveis Anos de Estudo e Hábitos de Leitura e Escrita, por Grupo

\begin{tabular}{lcccc}
\hline & \multicolumn{2}{c}{ Crianças $(n=35)$} & \multicolumn{2}{c}{ Adultos $(n=54)$} \\
\hline Tarefa de Escrita & Anos de estudo & Idade & Anos de estudo & Hábitos de L e E \\
\hline Palavras & $0,34^{*}$ & 0,28 & $0,42^{*}$ & $0,31^{*}$ \\
Regulares & $0,35^{*}$ & 0,20 & $0,29^{*}$ & $0,29^{*}$ \\
Irregulares & 0,25 & 0,28 & $0,39^{* *}$ & $0,27^{*}$ \\
Curtas & 0,29 & 0,21 & $0,40^{*}$ & 0,25 \\
Longas & 0,33 & 0,29 & $0,33^{*}$ & 0,19 \\
Frequentes & 0,10 & 0,10 & $-0,03$ & $-0,10$ \\
Não frequentes & $0,34^{*}$ & $0,35^{*}$ & $0,40^{* *}$ & $0,35^{* *}$ \\
Pseudopalavras & 0,29 & $0,40^{*}$ & $0,37^{* *}$ & 0,22 \\
Curtas & $0,34^{*}$ & 0,04 & $0,27^{*}$ & 0,25 \\
Longas & $-0,09$ & $0,40^{*}$ & $0,33^{*}$ & 0,19 \\
Total & $0,36^{*}$ & $0,45^{*}$ & $0,45^{*}$ & $0,29 *$ \\
\hline
\end{tabular}

$*=p<0,05 ; * *=p<0,01$.

Nas comparações entre os grupos não foram encontradas diferenças entre as frequências dos tipos de erros na TLPP $(p>0,05)$. De modo geral, os grupos tiveram de $87,5 \%$ a $100 \%$ de acertos no total da tarefa. No grupo das crianças verificou-se um efeito de extensão $(Z=4,484$; $p<0,001)$, assim como nos adultos com até 10 anos de estudo $(Z=3,968 ; p<0,001)$ e nos adultos com mais de 11 anos de escolaridade $(Z=$ 3,536; $p<0,001)$. O grupo de crianças também exibiu efeito de lexicalidade $(Z=5,027$; $p<$ $0,001)$, o que também foi identificado nos adultos com até 10 anos de estudo $(Z=4,385 ; p<$ $0,001)$ e de maior escolaridade $(Z=4,641,000$; $p<0,001)$. As crianças $(Z=2,178 ; p=0,029) \mathrm{e}$ os adultos com menor número de anos estuda$\operatorname{dos}(Z=3,097 ; p=0,002)$, mas não os adultos com alta escolaridade $(p=0,096)$, apresentaram também efeito de frequência. Além disso, como visto na Tabela 5, quando comparados os efeitos psicolinguísticos entre os grupos, o efeito de frequência foi o único que mostrou diferença estatisticamente significativa, em contraste com os outros tipos de efeito.

Em relação aos tipos de erros, apenas um adulto com até 10 anos de estudo apresentou um erro do tipo neologismo para leitura. Nenhum participante obteve erros do tipo paralexia semântica, semântico-fonológica, verbal e perseveração. Os tipos de erros encontrados na leitura dos participantes são apresentados na Tabela 6 .

Para fornecer dados preliminares da TLPP, a partir dos acertos dos grupos na leitura de palavras reais, regulares, irregulares, pseudopalavras e no total da tarefa, foram calculados os percentis correspondentes ao desempenho para a classificação dos participantes (Anexo B). Relacionando estes aos escores padronizados $(z), \mathrm{o}$ percentil 15 ou 16 (valores de escore $z$ de $-1,02$ a $-1,05)$ pode ser comparado a -1 desvio padrão (sugestivo de alerta para déficit), o percentil 7 (valores de escore $z$ de $-1,44$ a -1,51) equivale a $-1,5$ desvio padrão (sugestivo de déficit moderado a severo) e o percentil 2 a 2,5 é equivalente a -2 desvios padrão. Escores $z$ entre $-1,96$ a -2,19 são sugestivos de déficit de gravidade importante (Strauss, Sherman, \& Spreen, 2006). Portanto, uma criança com 21 acertos nas palavras irregulares, teria um desempenho deficitário em nível grave, enquanto outra criança com 23 acertos poderia ter um desempenho indicativo de déficit. 
Tabela 5

Desempenho (Média e Desvio-padrão) da Porcentagem de Acertos e de Erros nas Categorias de Estímulos da Tarefa de Leitura de Palavras/ Pseudopalavras e Resultado da Comparação entre Grupos

\begin{tabular}{|c|c|c|c|c|}
\hline & $\begin{array}{l}\text { Crianças }^{\mathrm{a}} \\
(n=35)\end{array}$ & $\begin{array}{l}\text { Adultos até } 10 \\
\text { anos de estudo } \\
\qquad(n=25)\end{array}$ & $\begin{array}{l}\text { Adultos - } 11 \text { ou mais } \\
\text { anos de estudo } \\
(n=29)\end{array}$ & \\
\hline Tarefa de Leitura & $M(D P)$ & $M(D P)$ & $M(D P)$ & $H$ \\
\hline Palavras Reais & $98,80(2,22)$ & $98,08(2,47)$ & $99,64(0,80)$ & $7,148 * \mathrm{bc}$ \\
\hline Regulares & $99,16(1,69)$ & $99,5(1,38)$ & $100(0)$ & $6,121 * a c$ \\
\hline Irregulares & $98,57(2,84)$ & $96,66(4,50)$ & $99,28(1,60)$ & $6,177 * b \mathrm{c}$ \\
\hline Curtas & $99,28(2,14)$ & $98,66(2,32)$ & $99,85(0,77)$ & $6,596 * \mathrm{bc}$ \\
\hline Longas & $98,33(2,71)$ & $97,5(3,80)$ & $99,42(1,46)$ & 4,720 \\
\hline Frequentes & $99,40(1,79)$ & $100(0)$ & $99,85(0,77)$ & 3,882 \\
\hline Não frequentes & $98,21(3,39)$ & $96,16(4,94)$ & $98,85(3,32)$ & $6,738 * b \mathrm{bc}$ \\
\hline Efeito de regularidade & $0,60(2,50)$ & $2,83(4,45)$ & $0,72(1,60)$ & 4,968 \\
\hline Efeito de extensão & $0,95(2,04)$ & $1,17(3,90)$ & $0,43(1,70)$ & 1,338 \\
\hline Efeito de frequência & $1,19(3,12)$ & $3,83(4,94)$ & $1,01(3,46)$ & $7,955^{* b c}$ \\
\hline Efeito de lexicalidade & $10,95(6,95)$ & $14,08(8,20)$ & $10,56(5,22)$ & 2,731 \\
\hline Pseudopalavras & $87,85(7,38)$ & $84,0(8,56)$ & $89,08(5,38)$ & 5,145 \\
\hline Curtas & $94,04(6,57)$ & $91,66(8,67)$ & $93,10(18,77)$ & 3,472 \\
\hline Longas & $80,47(10,68)$ & $76,33(11,70)$ & $81,60(9,28)$ & 2,973 \\
\hline Efeito de extensão & $13,57(11,09)$ & $15,33(11,46)$ & $11,49(19,84)$ & 0,596 \\
\hline Total de Acertos & $95,15(3,27)$ & $93,38(3,68)$ & $96,12(2,01)$ & $7,647 * b \mathrm{bc}$ \\
\hline Total de Erros & $11,80(8,72)$ & $16,40(13,26)$ & $8,69(7,66)$ & $8,022 * b c$ \\
\hline Tipos de erros & $8,2(5,32)$ & $10,88(7,14)$ & $6,0(4,35)$ & $9,812 * \mathrm{bc}$ \\
\hline
\end{tabular}

\section{Discussão}

O presente trabalho apresentou o processo de construção da TLPP, bem como o desempenho de crianças e adultos na tarefa, tanto para justificar a adequação dos itens desta tarefa, como para contribuir com dados para clínicos e pesquisadores que desejam incluí-la na avaliação da leitura de palavras. As análises de juízes mostraram-se importantes para garantir que os estímulos da TLPP contemplavam os critérios psicolinguísticos para além das listas de pala- vras. Outras investigações que utilizaram estímulos linguísticos e demonstraram as etapas de adaptação ou desenvolvimento de instrumentos também ressaltaram a importância de controlar as características dos itens para uma adequada avaliação neuropsicológica da linguagem (Fontoura, Rodrigues, Parente, Fonseca, \& Salles, 2011; Lúcio et al., 2012; Rodrigues \& Salles, 2013). Espera-se que a descrição detalhada dessa tarefa possa nortear outros estudos que se propõem a seguir a abordagem da neuropsicologia cognitiva para criar ferramentas de avaliação. 
Tabela 6

Frequência e Porcentagem do Número de Erros Identificados na Leitura, por Grupo

\begin{tabular}{lccc}
\hline & $\begin{array}{c}\text { Crianças } \\
(n=35)\end{array}$ & $\begin{array}{c}\text { Adultos até } 10 \text { anos } \\
\text { de estudo } \\
(n=25)\end{array}$ & $\begin{array}{c}\text { Adultos - 11 ou mais } \\
\text { anos de estudo } \\
(n=29)\end{array}$ \\
\hline Erros em Leitura & $F(\%)$ & $F(\%)$ & $F(\%)$ \\
Paralexia fonológica & $1(0,59)$ & $3(1,75)$ & $4(3,39)$ \\
Paralexia morfêmica & $4(2,39)$ & $1(0,58)$ & $1(0,85)$ \\
Desconsideração de regra & $40(23,81)$ & $36(21,05)$ & $38(32,20)$ \\
Regularização & $9(5,36)$ & $8(4,68)$ & $1(0,85)$ \\
Acentuação & $46(27,38)$ & $22(12,87)$ & $19(16,10)$ \\
Lexicalização & $6(3,57)$ & $10(5,85)$ & $4(3,39)$ \\
Substituição & $32(19,05)$ & $41(23,98)$ & $26(22,03)$ \\
Omissão & $10(5,95)$ & $17(9,94)$ & $4(3,39)$ \\
Acréscimo & $12(7,14)$ & $18(10,53)$ & $10(8,48)$ \\
Transposição & $8(4,76)$ & $14(8,19)$ & $11(9,32)$ \\
\hline Total & $168(100)$ & $171(100)$ & $118(100)$ \\
\hline
\end{tabular}

$\mathrm{Na}$ avaliação da leitura, de acordo com o modelo de dupla-rota, espera-se maior acurácia na leitura de palavras reais, curtas, regulares e frequentes, em relação às pseudopalavras, palavras longas, irregulares e não frequentes (Salles $\&$ Parente, 2007). Nesse aspecto, foi observado que a TLPP seguiu o padrão sugerido. Além disso, a tarefa mostrou-se adequada para diferenciar leitores de baixa e alta escolaridade. Uma possibilidade para estudos futuros é a avaliação das propriedades psicométricas da tarefa, conforme sugerem Lúcio et al. (2012) e Lúcio e Pinheiro (2013). Para tanto, seria necessário o aumento da amostra e uma divisão dos adultos por variações menores de anos de estudo e de hábitos de leitura e escrita, uma vez que foi identificada uma relação dessas variáveis com a porcentagem de acertos na TLPP.

Nas análises iniciais de correlação, no grupo de crianças identificou-se que quanto maior a idade e o número de anos estudados, melhor o desempenho na leitura de palavras reais e regulares e na leitura de palavras não frequentes e pseudopalavras longas. Esses achados corroboram o estudo de Salles e Parente (2007), que verificou que crianças entre sete e 13 anos de idade utilizam as rotas lexical e fonológica na leitura de palavras. A relação entre as variáveis idade e anos de estudo já era esperada na amostra de crianças, pois todas estavam regularmente matriculadas e não apresentavam histórico de repetência escolar. De acordo com Salles et al. (2013), idade e escolaridade são variáveis inter-relacionadas que contribuem de forma complementar no aperfeiçoamento da leitura. A variável QI não apresentou relação com o desempenho na leitura de palavras e pseudopalavras, o que também era esperado, dado que testes de inteligência mostram-se ineficientes para prever sucesso na aquisição desta habilidade (Kortteinen, Närhi, \& Ahonen, 2009; Maia \& Fonseca, 2002; Salles, Zamo, Rodrigues, \& Jou, 2010), ainda mais em crianças com anos de estudo mais avançados.

Em relação aos adultos, verificou-se correlação positiva entre escolaridade e quase todas as medidas da TLPP, bem como entre hábitos de leitura e escrita e os escores nas palavras reais (regulares e irregulares), não frequentes, e no total da tarefa. Esses dados corroboram a importância da escolaridade para o desempenho de adultos na leitura de palavras (Pawlowski, Fonseca, Salles, Parente, \& Bandeira, 2008), assim 
como já foi demonstrado para a escrita (Pawlowski et al., 2012; Rodrigues, 2013). As palavras retiradas das listas classificadas por frequência são extraídas de materiais escritos, o que pode ter facilitado o melhor desempenho dos participantes que têm maiores hábitos de leitura e escrita na TLPP. Assim, a alta escolaridade e os altos hábitos de leitura e escrita podem contribuir para a aquisição das regras ortográficas e assim influenciar positivamente o desempenho de adultos em tarefas linguísticas (Lecours \& Parente, 1997; Pawlowski et al., 2012). O envelhecimento não parece estar relacionado com prejuízo na leitura de palavras em adultos, mas somente quanto à velocidade de leitura e à compreensão textual (Carthery-Goulart \& Parente, 2006).

De modo geral, os participantes desse estudo, todos leitores proficientes, obtiveram mais de $85 \%$ de acertos na TLPP, desempenho também encontrado em outras pesquisas brasileiras (Salles \& Parente, 2007; Salles et al., 2013). Uma vez que se aceitam autocorreções durante a execução da tarefa de leitura é comum ocorrerem altos escores. Assim, para estudos futuros, sugere-se o uso de medidas de velocidade de leitura e tempo de reação, que poderiam melhor diferenciar a precisão dessa habilidade, conforme demonstrado por Christopher et al. (2012).

Dentro dos grupos (crianças e adultos) observaram-se efeitos de extensão, frequência, regularidade e lexicalidade, o que era esperado de acordo com o modelo de dupla-rota. Efeitos de frequência e lexicalidade podem indicar que os participantes apresentaram melhores escores nos estímulos prioritariamente lidos pela rota lexical, que se torna mais automatizada, conforme o desenvolvimento do aprendizado de leitura (Lúcio \& Pinheiro, 2011). Os efeitos de extensão e regularidade sugerem uma leitura que se utiliza principalmente da rota fonológica (Salles \& Parente, 2007). Portanto, pode-se considerar também que a TLPP é sensível aos efeitos psicolinguísticos que indicam uso preferencial de rota para leitura de palavras e pseudopalavras.

$\mathrm{Na}$ comparação entre os grupos, observou-se que os adultos com menor escolaridade exibiram um desempenho inferior tanto ao grupo de crianças quanto ao de adultos com maior número de anos de estudo nas medidas da TLPP. Esse resultado reforça a importância da escolaridade para a habilidade de leitura e conhecimento das regras ortográficas (Lecours \& Parente, 1997), além de indicar a sensibilidade do instrumento para diferenciar esses grupos. Ainda, os adultos com menor escolaridade apresentaram maior número de erros e de diferentes tipos de erros em comparação com adultos com 11 ou mais anos de estudo. Isso ocorreu principalmente para as palavras não frequentes, além de o efeito de frequência ser mais acentuado nos adultos com até 10 anos de estudo. Esses achados corroboram dados do trabalho de Tainturier, Tremblay e Lecours (1992), que identificaram em uma tarefa de decisão lexical que o efeito de frequência das palavras (desempenho superior nas palavras frequentes, em relação às não frequentes) é sensível à duração dos anos de escolaridade, sendo mais acentuado nos indivíduos menos escolarizados. É possível que os participantes mais escolarizados tenham representações mais consistentes no léxico (memória das palavras), independentemente de ser a palavra frequente ou não na linguagem escrita.

Na classificação dos tipos de erros, não foram encontradas diferenças significativas entre os grupos, sendo observados mais frequentemente na amostra os erros de desconsideração de regra contextual, acentuação e substituição de letras nas palavras, indicando uma estratégia fonológica de leitura. O baixo número de erros pode ter ocorrido pelo fato de a leitura de palavras já ter sido automatizada nos participantes. Ainda, um maior número de erros do tipo regra, acentuação e substituição de letras pode ter ocorrido pelas constantes mudanças das normas ortográficas da língua portuguesa, o que também foi observado na avaliação da escrita de palavras em adultos (Rodrigues, Pawlowski, Müller, Bandeira, \& Salles, 2013).

Não foram encontrados na amostra os erros do tipo paralexia semântica, semântico-fonológica, verbal e perseveração, além de somente um adulto de baixa escolaridade ter cometido o erro do tipo neologismo na leitura de uma pseudopalavra. Ler uma palavra no lugar de outra, geralmente, ocorre em indivíduos que sofreram 
algum tipo de dano cerebral e que apresentam dificuldade em acessar as rotas (lexical e/ ou fonológica) para a leitura de palavras (Ellis, 1995). Assim, sugere-se que esses tipos de erros possam diferenciar casos clínicos de controles na avaliação neuropsicológica dessa habilidade. $\mathrm{O}$ neologismo talvez tenha sido verbalizado por esse participante por uma dificuldade na conversão dos grafemas em fonemas para a leitura das palavras, uma vez que esse tipo de resposta não pode estar armazenado no léxico (Temple, 1997), o que também pode ser observado em crianças nas séries iniciais (Pinheiro \& Rothe-Neves, 2001; Salles \& Parente, 2007).

Os dados preliminares apresentados em percentis mostram novamente altos escores na leitura de palavras, quando se aceitam autocorreções, embora leitores proficientes apresentem poucos erros como observado no desempenho dos grupos. Ressalta-se que para uma avaliação completa da leitura é importante a inclusão de tarefas com frases e textos (simples e complexos), além de avaliar processos como velocidade de leitura e de compreensão textual. Assim, pode-se ter um perfil mais preciso das dificuldades linguísticas de cada paciente.

\section{Conclusão}

Tarefas que se propõem a avaliar a leitura de palavras e pseudopalavras, seguindo o modelo teórico de dupla-rota, devem garantir que os estímulos incluídos contemplem os critérios psicolinguísticos, tais como regularidade, lexicalidade, extensão e frequência, a fim de verificar os aspectos cognitivos envolvidos nesse processamento. Além disso, devem ser considerados os tipos de erros, que são indicativos de perfis de déficits. Espera-se que a apresentação criteriosa do processo de construção de uma tarefa de leitura contribua com demais pesquisadores que utilizam estímulos linguísticos na adaptação e construção de instrumentos.

Ainda, os dados preliminares aqui apresentados, escassos no Brasil, podem auxiliar clínicos e profissionais que trabalham nas escolas que desejam utilizar essa tarefa em uma avaliação mais aprofundada da leitura de palavras. No entanto, ressalta-se como limitação a amostra ser composta apenas por crianças de escolas particulares e os adultos contemplarem apenas uma faixa etária. Além disso, espera-se que o presente artigo contribua para o estudo do desempenho em leitura em adultos e crianças mais velhas, que são tradicionalmente menos estudados que crianças em fase de alfabetização. Estudos futuros podem ser direcionados a mostrar o desempenho de casos clínicos na TLPP.

$\mathrm{Na}$ avaliação de leitura de palavras e pseudopalavras de adultos é importante considerar suas características sociodemográficas. Para tanto, é necessário realizar estudos brasileiros com adultos de diferentes faixas de idade, escolaridade e hábitos de leitura e escrita, identificando as frequências de erros, acertos, tipos de erros e efeitos psicolinguísticos na TLPP. Dessa forma, poderiam ser obtidos pontos de cortes adequados para cada grupo de participantes nessas variáveis e melhor identificar os pacientes que realmente apresentam uma dislexia.

\section{Referências}

Angelini, A. L., Alves, I. C. B., Custódio, E. M., Duarte, E. F., \& Duarte, J. L. M. (1999). Matrizes Progressivas Coloridas de Raven: Escala especial. Manual. São Paulo, SP: Centro Editor de Testes e Pesquisa em Psicologia.

Beck, A. T., Steer, R., \& Brown, G. (1996). BDIII: Beck Depression inventory-II Manual. New York: The Psychological Corporation.

Capovilla, A. G. S., \& Capovilla, F. C. (1998). Desenvolvimento de leitura e escrita de pré-3 a 2a. série: Tabelas preliminares de normatização de uma lista de itens psicolinguísticos. Ciência Cognitiva: Teoria, Pesquisa e Aplicação, 2(4), 821-840.

Capovilla, F. C., Capovilla, A. G. S., \& Macedo, E. C. (1998). Validação do software_CronoFonos para análise de tempo de reação, duração e frequência de segmentação locucionais na leitura em voz alta de itens isolados. Ciência Cognitiva: Teoria, Pesquisa e Aplicação, 2(3), 253-340.

Capovilla, F. C., Varanda, C., \& Capovilla, A. G. S. (2006). Teste de Competência de Leitura de Palavras e Pseudopalavras: Normatização e validação. Psicologia: Revista da Vetor Editora, 7(2), 47-59. 
Carthery-Goulart, M. T., \& Parente, M. A. M. P. (2006). Leitura e escrita e o envelhecimento. In M. A. M. P. Parente (Ed.), Cognição e envelhecimento (pp. 191-202). Porto Alegre, RS: Artmed.

Chaves, M. L., \& Izquierdo, I. (1992). Differential diagnosis between dementia and depression: A study of efficiency increment. Acta Neurologica Scandinavia, 11, 412-429.

Christopher, M. E., Miyake, A., Keenan, J. M., Pennington, B., DeFries, J. C., Wadsworth, S. J., ... Olson, R. K. (2012). Predicting word reading and comprehension with executive function and speed measures across development: A latent variable analysis. Journal of Experimental Psychology. General, 141(3), 470-488. doi:10.1037/ a0027375

Coltheart, M. (2006). Dual route and connectionist models of reading: An overview. London Review of Education, 4(1), 5-17. doi:10.1080/13603110600574322.

Ellis, A. W. (1995). Leitura, escrita, dislexia. Uma análise cognitiva. Porto Alegre, RS: Artes Médicas.

Fontoura, D. R., Rodrigues, J. C., Parente, M. A. M. P., Fonseca, R. P., \& Salles, J. F. (2011). Adaptação do Instrumento de Avaliação Neuropsicológica Breve NEUPSILIN para avaliar pacientes com afasia expressiva: NEUPSILIN-Af. Ciências \& Cognição, 16(3), 78-94.

Gorenstein, C., Pang, W. Y., Argimon, I. L., \& Werlang, B. S. G. (2011). BDI-II - Inventário de depressão de Beck. Porto Alegre, RS: Casa do Psicólogo.

Gutierrez, L., \& Tomasi, E. (2011). Prevalência de dislexia e fatores associados do $1^{\circ}$ ao $4^{\circ}$ ano. Trabalho apresentado no XIII Encontro de Pós-Graduação da Universidade Federal de Pelotas, RS, Brasil.

Janczura, G. A., Castilho, G. M., Rocha, N. O., Van Erven, T. J. C., \& Huang, T. P. (2007). Normas de concretude para 909 palavras da língua portuguesa. Psicologia: Teoria e Pesquisa, 23, $195-$ 204.

Kochhann, R., Varela, J. S., Lisboa, C. S. M., \& Chaves, M. L. F. (2010). The Mini Mental State Examination: Review of cutoff points adjusted for schooling in a large Southern Brazilian sample. Dementia \& Neuropsychologia, 4(1), 35-41.
Kortteinen, H., Närhi, V., \& Ahonen, T. (2009). Does IQ matter in adolescents' reading disability? Learning and Individual Differences, 19, $257-$ 261.

Lecours, A. R., \& Parente, M. A. M. P. (1997). Dislexia: Implicações do sistema de escrita do português. São Paulo, SP: Artes Médicas.

Lúcio, P. S., Moura, R. J. de, Nascimento, E. do, \& Pinheiro, A. M. V. (2012). The construction of a word reading aloud task: A psychometric item analysis report. Psicologia: Reflexão $e$ Crítica, 25(4), 662-670. doi:10.1590/S010279722012000400005

Lúcio, P. S., \& Pinheiro, A. M. V. (2011). Vinte anos de estudo sobre o reconhecimento de palavras em crianças falantes do português: Uma revisão de literatura. Psicologia: Reflexão $e$ Crítica, 24(1), 170-179. doi:10.1590/S010279722011000100020

Lúcio, P. S., \& Pinheiro, A. M. V. (2013). Escala da Avaliação da Competência da Leitura pelo Professor (EACOL): Evidências de validade de critério. Temas em Psicologia, 21(2), 499-511. doi:10.9788/TP2013.2-15

Maia, A. C. B., \& Fonseca, M. L. (2002). Quociente de inteligência e aquisição de leitura: Um estudo correlacional. Psicologia: Reflexão e Crítica, 15(2), 261-270. doi:10.1590/S010279722002000200004

Pawlowski, J., Fonseca, R. P., Salles, J. F., Parente, M. A. M. P., \& Bandeira, D. R. (2008). Evidências de validade do Instrumento de Avaliação Neuropsicológica Breve Neupsilin. Arquivos Brasileiros de Psicologia, 60(2), 101-116.

Pawlowski, J., Remor, E., Parente, M. A. M., Salles, J. F., Fonseca, R. P., \& Bandeira, D. R. (2012). The influence of reading and writing habits associated with education on the neuropsychological performance of Brazilian adults. Reading and Writing, 25, 2275-2289.

Pinheiro, A. M. V. (2003). Avaliação cognitiva das capacidades de leitura e de escrita de crianças nas séries iniciais do ensino fundamental - AVACLE: Relatório Final Global e Integrado de atividades desenvolvidas, submetido ao Conselho Nacional de Desenvolvimento Cientifico e Tecnológico (Processo 52089/93-0). Belo Horizonte, MG: Universidade Federal de Minas Gerais, Departamento de Psicologia. 
Pinheiro, A. M. V., Cunha, C. R. da, \& Lúcio, P. S. (2008). Tarefa de leitura de palavras em voz alta: Uma proposta de análise dos erros. Revista Portuguesa de Educação, 21(2), 115-138.

Pinheiro, A. M. V., \& Rothe-Neves, R. (2001). Avaliação cognitiva de leitura e escrita: As tarefas de leitura em voz alta e ditado. Psicologia: Reflexão e Crítica, 14(2), 399-408. doi:10.1590/ S0102-79722001000200014

Pritchard, S. C., Coltheart, M., Palethorpe, S., \& Castles, A. (2012). Nonword reading: Comparing dual-route cascaded and connectionist dualprocess models with human data. Journal of Experimental Psychology. Human Perception and Performance, 38(5), 1268-1288. doi:10.1037/ a0026703.

Rodrigues, J. C. (2013). Análise cognitiva da escrita de palavras de adultos após acidente vascular cerebral nos hemisférios direito e esquerdo (Dissertação de mestrado, Instituto de Psicologia, Universidade Federal do Rio Grande do Sul, Porto Alegre, RS, Brasil).

Rodrigues, J. C., Pawlowski, J., Müller, J. L., Bandeira, D. R., \& Salles, J. F. (2013). Comparação dos tipos de erros na escrita de palavras entre adultos após AVC unilateral nos hemisférios direito e esquerdo. Revista Neuropsicologia Latinoamerica, 5(4).

Rodrigues, J. C., \& Salles, J. F. (2013). Tarefa de escrita de palavras/ pseudopalavras para adultos: Abordagem da neuropsicologia cognitiva. Letras de Hoje - Estudos e Debates de Assuntos de Linguística, Literatura e Língua Portuguesa, 48(1), 50-58.

Salles, J. F., \& Parente, M. A. M. P. (2002). Processos cognitivos na leitura de palavras em crianças: Relações com compreensão e tempo de leitura. Psicologia: Reflexão e Crítica, 15, 321-331.

Salles, J. F., \& Parente, M. A. M. P. (2004). Compreensão textual em alunos de segunda e terceira séries: Uma abordagem cognitiva. Estudos de Psicologia (Natal), 9, 71-80.

Salles, J. F., \& Parente, M. A. M. P. (2007). Avaliação da leitura e escrita de palavras em crianças de $2^{\mathrm{a}}$ série: Abordagem neuropsicológica cognitiva. Psicologia Reflexão \& Crítica, 20(2), 220228. doi:10.1590/S0102-79722007000200007
Salles, J. F., Piccolo, L. R., Zamo, R. S., Toazza, R. (2013). Normas de desempenho em tarefa de leitura de palavras/pseudopalavras isoladas (LPI) para crianças de $1^{\circ}$ ano a $7^{\circ}$ ano. Estudos e Pesquisas em Psicologia, 13(2), 397-419.

Salles, J. F., Zamo, R. S., Rodrigues, J. C., \& Jou, G. I. (2010). Dificuldades de leitura e escrita: Revisão sobre os critérios de avaliação e identificação. In S. R. K. Guimarães \& M. R. Maluf (Eds.), Aprendizagem da linguagem escrita (pp. 33-60). São Paulo, SP: Vetor.

Sardinha, T. B. (2003). The Bank of Portuguese. Direct Papers, 50. Retrieved from http://www2. lael.pucsp.br/ tony/tony/pesquisa.html

Sinanović, O., Mrkonjić, Z., Zukić, S., Vidović, M., \& Imamović, K. (2011). Post-stroke language disorders. Acta Clinica Croatica, 50(1), 79-94.

Strauss, E., Sherman, E. M. S., \& Spreen, O. (2006). A compendium of neuropsychological tests: Administration, norms and commentary. New York: Oxford University Press.

Tainturier, M. J., Tremblay, M., \& Lecours, A. R. (1992). Educational level and the word frequency effect: A lexical decision investigation. Brain and Language, 43, 460-474.

Temple, C. (1997). Developmental cognitive neuropsychology brain damage, behavior, and cognitive series. London: Psychology Press.

Thambirajah, M. S. (2010). Developmental dyslexia: An overview. Advances in Psychiatric Treatment, 16(4), 299-307. doi:10.1192/apt. bp. 108.006072
Recebido: 25/02/2014

$1^{a}$ revisão: 06/08/2014 Aceite final: 02/09/2014 


\section{Anexo A}

\section{Tarefa de Leitura de Palavras e Pseudopalavras}

\section{Materiais:}

\section{A) Leitura em voz alta}

- Gravador digital

- Livro de estímulos com as palavras escritas

Instruções: Deve ser apresentado um estímulo por vez, centralizado à mesa, à frente do participante. Essa tarefa deverá ser gravada e registrada, para posterior análise dos tipos de erros de leitura.

- Para palavras: vou lhe mostrar algumas palavras e você deve ler elas em voz alta, da maneira que achar melhor. Podemos começar?

\begin{tabular}{lllll}
\hline 1. Carta & 11.Barba & 21. Chinelo & 31. Caderno & 41. Cárie \\
2. Janela & 12. Jaula & 22. Inseto & 32. Gola & 42. Escola \\
3. Amarelo & 13. Farelo & 23. Taxímetro & 33. Terra & 43. Terno \\
4. Lesma & 14. Tosse & 24. Grade & 34. Saxofone & 44. Futebol \\
5. Garra & 15. Droga & 25. Meia & 35. Criança & 45. Cama \\
6. Felino & 16. Jovem & 26. Acerola & 36. Rede & 46. Exército \\
7. Correnteza & 17. Selva & 27. Gosma & 37. Machucado & 47. Família \\
8. Picada & 18. Torta & 28. Sexo & 38. Festa & 48. Comida \\
9. Dinheiro & 19. Rosa & 29. Fermento & 39. Transporte & \\
10. Cidade & 20. Tabaco & 30. Leite & 40. Filho & \\
\hline
\end{tabular}

- Para pseudopalavras: Agora vou lhe mostrar palavras que não existem (não tem significado), mas você deve ler todas elas da maneira que achar melhor. Podemos começar?

$\begin{array}{lllll}\text { 1. Teile } & \text { 6. Mesla } & \text { 11. Maralo } & \text { 16. Bolefu } & \text { 21. Fosaxone } \\ \text { 2. Rorola } & \text { 7. Chonile } & \text { 12. Bafau } & \text { 17. Senjo } & \text { 22. Zareo } \\ \text { 3. Gadra } & \text { 8. Lajau } & \text { 13. Tisso } & \text { 18. Nurto } & \text { 23. Etixero } \\ \text { 4. Jenala } & \text { 9. Taspobe } & \text { 14. Zarronte } & \text { 19. Tilhu } & \text { 24. Cavermo } \\ \text { 5. Varte } & \text { 10. Tomenfo } & \text { 15. Moxe } & \text { 20. Divairo } & \end{array}$




\section{Anexo B}

Percentis de Acertos por Escore e por Grupo

\begin{tabular}{|c|c|c|c|c|c|}
\hline \multicolumn{6}{|c|}{ Crianças $(M=10,80 ; D P=0,83$ anos $)(n=35)$} \\
\hline Percentil & Palavras & Regulares & Irregulares & Pseudopalavras & Total \\
\hline 2,5 & 43 & 23 & 21 & 18 & 63 \\
\hline 7 & 45,52 & 23 & 22,52 & 18 & 64,52 \\
\hline 10 & 46 & 23 & 23 & 18,60 & 65 \\
\hline 16 & 47 & 23 & 23 & 19 & 66 \\
\hline 20 & 47 & 23,20 & 23 & 19 & 66 \\
\hline 30 & 47 & 24 & 24 & 20 & 67 \\
\hline 40 & 48 & 24 & 24 & 20 & 68 \\
\hline 50 & 48 & 24 & 24 & 22 & 69 \\
\hline 60 & 48 & 24 & 24 & 22 & 70 \\
\hline 70 & 48 & 24 & 24 & 22 & 70 \\
\hline 80 & 48 & 24 & 24 & 23 & 70,80 \\
\hline 90 & 48 & 24 & 24 & 23 & 71 \\
\hline \multicolumn{6}{|c|}{ Adultos $(M=54,80 ; D P=10,15$ anos $)$ até 10 anos de estudo $(n=25)$} \\
\hline Percentil & Palavras & Regulares & Irregulares & Pseudopalavras & Total \\
\hline 2,5 & 44 & 23 & 21 & 16 & 63 \\
\hline 7 & 44,82 & 23 & 21 & 16,82 & 63 \\
\hline 10 & 45 & 23 & 21 & 17 & 63 \\
\hline 16 & 46 & 24 & 22 & 18 & 64 \\
\hline 20 & 46 & 24 & 22 & 18 & 64,20 \\
\hline 30 & 46,80 & 24 & 23 & 19 & 65,80 \\
\hline 40 & 47 & 24 & 23 & 19,40 & 66 \\
\hline 50 & 48 & 24 & 24 & 21 & 68 \\
\hline 60 & 48 & 24 & 24 & 21 & 69 \\
\hline 70 & 48 & 24 & 24 & 22 & 69 \\
\hline 80 & 48 & 24 & 24 & 22 & 69,80 \\
\hline 90 & 48 & 24 & 24 & 23 & 71 \\
\hline \multicolumn{6}{|c|}{ Adultos $(M=52,62 ; D P=8,93$ anos $)-11$ ou mais anos de estudo $(n=29)$} \\
\hline Percentil & Palavras & Regulares & Irregulares & Pseudopalavras & Total \\
\hline 2,5 & 47 & 24 & 23 & 19 & 66 \\
\hline 7 & 47 & 24 & 23 & 19,03 & 67 \\
\hline 10 & 47 & 24 & 23 & 19,90 & 67 \\
\hline 16 & 47 & 24 & 23 & 20 & 67,64 \\
\hline 20 & 47,80 & 24 & 23,80 & 20 & 68 \\
\hline 30 & 48 & 24 & 24 & 20,70 & 68 \\
\hline 40 & 48 & 24 & 24 & 21 & 69 \\
\hline 50 & 48 & 24 & 24 & 21 & 69 \\
\hline 60 & 48 & 24 & 24 & 22 & 70 \\
\hline 70 & 48 & 24 & 24 & 22,30 & 70 \\
\hline 80 & 48 & 24 & 24 & 23 & 71 \\
\hline 90 & 48 & 24 & 24 & 23 & 71 \\
\hline
\end{tabular}

